\title{
Error Augmentation Feedback for Lateral Weight Shifting
}

\author{
Kevin O'Brien ${ }^{\mathrm{a}, *}$, Charles R. Crowell ${ }^{\mathrm{b}}$, James Schmiedeler ${ }^{\mathrm{a}}$ \\ ${ }^{a}$ Department of Aerospace and Mechanical Engineering, University of Notre Dame, Notre Dame, IN 46556 \\ ${ }^{b}$ Department of Psychology, University of Notre Dame, Notre Dame, IN 46556
}

\begin{abstract}
This study examines the effect of error augmentation of center of pressure $(\mathrm{CoP})$ visual feedback on the performance of a lateral weight shifting task. Error augmentation emphasizes deviations from a standard CoP trajectory generated from existing data of over 2000 weight shifts collected with young, healthy subjects. Thirty-six subjects completed nine lateral weight shifting sessions, of which four were training sessions between each of the five testing sessions. Half of the subjects received error augmentation feedback during the training sessions, while the other half received the unaltered, control feedback. The change in visual feedback did not affect the final steady state weight shifting performance. Instead, error augmentation feedback was found to drive subjects to their steady-state performance sooner than unaltered visual feedback. The emphasis on deviations from the standard trajectory with error augmentation appears to lead to reduced variation in shifting. This finding may be useful in generating novel therapies that improve the efficiency of balance rehabilitation. Keywords: Lateral Balance, Visual Feedback, Error Augmentation, Center of Pressure, Time-To-Target
\end{abstract}

\section{Introduction}

Visual, vestibular, and somatosensory feedback all contribute to maintaining balance while standing upright [1]. Visual feedback in particular is useful for evaluating balance performance and can improve static balance through use of a force plate $[2,3]$. Functional balance, though, often involves tasks beyond quiet standing [4], such as weight transfer between legs in walking. This motivates analyzing balance performance during lateral weight shifting tasks, wherein visual

\footnotetext{
*Corresponding Author: kobrie23@nd.edu, (574) 631-xxxx

Email addresses: kobrie23@nd.edu (Kevin O'Brien), ccrowell@nd.edu (Charles R. Crowell), jschmie1@nd.edu (James Schmiedeler)
} 
feedback is critical for shifting weight to specified targets.

Much research has shown that feedback in simple motor training tasks improves performance, but less work has sought the optimal feedback within individual tasks [5]. There is evidence, though, that altering visual feedback affects balance performance [6, 7]. In arm reaching tasks, augmenting the visual feedback position error promotes temporary performance effects for new tasks [8, 9]. Error was measured as the distance from a straight-line path between the starting point and target and was visually augmented to encourage elimination of deviations from this path. Error augmentation helped speed subjects' adaptation to a rotation in the visual feedback [8, 9]. The effects of altering biofeedback in this manner are unknown for mobility and balance tasks [10]. Application of error augmentation to quiet standing balance has been inconclusive [11, 12], requiring large augmentation to improve performance. Effects on lateral weight shifting may be more pronounced, though, since it is typically more difficult and relies more heavily on visual feedback.

A primary metric in dynamic balance is time-to-target - the time required for the center of pressure $(\mathrm{CoP})$ feedback to move to the target [13]. Therefore, error augmentation must consider CoP motion over time, as opposed to previous methods in static balance that depend entirely on spatial CoP parameters. Most weight shifting research emphasizes controlled shifts because of their functional utility for walking and preventing falls. This study constrained shifts in two ways that restricted the speed of shifting - a shift could not be initiated until a new target appeared and the CoP marker had to remain in the target for a specified duration after the shift.

This paper aims to determine whether error augmentation in visual feedback can improve balance performance during a relatively simple, visually guided lateral weight shifting task. In arm reaching experiments, error augmentation promoted temporary performance effects for new tasks, but not improved overall steady state performance [14]. Hypothesizing that error argumentation offers the same benefits here as in arm reaching, we expect subjects presented with augmented error feedback to exhibit temporary performance improvement but reach the same steady state level of balance performance. 


\section{Methods}

\section{Subjects}

Thirty-six healthy subjects, 13 males and 23 females, ages 17 to 21 (18.9 \pm 1.0$)$ years, participated in this study. All subjects (or guardians) gave their informed consent prior to the study, and this research received approval from the appropriate Institutional Review Board. Evaluating the effects of augmented error feedback on weight shifting tasks required an experiment duration of roughly 40 minutes. To avoid fatigue effects, exclusively young, healthy subjects were recruited.

\section{Experimental System}

The WeHab system [15] employs an open-source program that uses the Nintendo Wii Balance Board to provide biofeedback during standard balance sessions. The system functions by connecting, via Bluetooth, one or two balance boards to a computer running the custom software. The position of the $\mathrm{CoP}$ of an individual standing on the balance board(s) is calculated from the force data and displayed as a green circle on an LCD screen (Figure 1). The balance board has been shown to perform comparably to sophisticated force plates for balance biofeedback $[16,17]$.

As shown in Figure 1, two balance boards were placed side-by-side to isolate each foot. Each subject stood with one foot centered on each board, facing a 27" monitor that displayed the 2dimensional position of the subject's CoP over an image of the balance board, as in [6]. The target was displayed as a vertical rectangle in a center, left, or right location. The center location corresponded to a 50-50 percent weight distribution, while the left and right locations corresponded to 70-30 percent weight distributions. A chair placed behind the subject allowed for a short rest between each session. The researcher sat nearby to run the software and ensure that the subject followed the protocol.

\section{Error Augmentation Design}

For lateral weight shifting, the standard CoP trajectory is unlikely to be a monotonic progression along a straight line. The CoP typically exhibits a non-minimum phase (NMP) behavior, moving away from the target due to the initial forces that ultimately drive it toward the target [18]. Therefore, the error must be calculated relative to a time trajectory of the CoP position, 
whereas for error augmentation in reaching [8], a time-independent path is sufficient. Additionally, it is beneficial to display the anterior-posterior movements of the CoP even when they are not relevant to the task [6]. Since it is not task-relevant, though, no error augmentation was applied in the anterior-posterior direction. A standard CoP time trajectory was generated by averaging data from 2287 shifts made by 27 young, healthy subjects [6] (Figure 2A). The lateral CoP trajectory does not vary with left/right direction, so these results average all shifts. There is little variation across the interquartile trajectories, particularly in the subjects' reaction time from when a target appears until the CoP first moves away from that target in its initial NMP behavior. The standard shift generated from previous studies may be different from those of a new subject pool, but it is reasonable to use a standard CoP trajectory that most young, healthy subjects employ, including the relatively static portion during the reaction time due to the small amount of variability for most shifts.

A subject's instantaneous error was computed as the difference between the true CoP position and the standard CoP position at the same instant. (The standard trajectory was taken to have a constant value for all times larger than the average shift duration.) The medial-lateral CoP position displayed was the actual position with the augmented error, equal to 0.5 times the true error, added to it (Figure 2B). The gain of 0.5 was tuned through pilot testing, which showed that small gains had too little effect on the feedback to be apparent to subjects and very large gains resulted in the type of excessive feedback manipulation known to reduce subjects' reliance on it [19]. With this error augmentation, if the CoP's lateral motion lagged the standard trajectory, the displayed CoP appeared to move more slowly than the actual CoP truly did, resulting in position lag. Similarly, if the actual CoP led the standard trajectory, the feedback showed the CoP to be moving even faster than it truly was, introducing overshoot of the true CoP position in the feedback.

\section{Experimental Protocol}

Subjects were randomly assigned to one of two equally sized groups: error augmentation and control. All subjects completed nine 3-minute balance sessions that all involved shifting their weight so as to move the displayed $\mathrm{CoP}$ indicator into vertically oriented, rectangular targets displayed on the screen (see Figure 1). Every second target appeared randomly to the left or right of center. 
For young, healthy subjects, there is no difference between left and right shifts, and randomly varying the shift direction was intended to reduce anticipation effects. As such, shifts to an offset target in either direction were considered for analysis. Subjects then shifted their displayed CoP back to a center target between shifts to each offset target. This process of alternatingly shifting the displayed $\mathrm{CoP}$ indicator into the offset rectangular targets and back to the central rectangular target was identical across every session. Subjects were instructed to hit as many targets as possible during the session, with the caveat that they needed to keep the CoP indicator in the target for approximately three seconds before the next target would appear. The actual required hold time was randomly varied between 2.5 and 3.5 seconds to reduce the subject's ability to anticipate the next target and begin shifting early. If the subject's CoP indicator left the target before this time limit, he/she needed to shift it back into the target for the entire hold time again. Following every balance session, subjects sat in the chair located behind the balance boards for 30 seconds of rest. Each experiment lasted approximately 40 minutes, including rest. Of the nine sessions, the odd sessions functioned as testing sessions, while the even sessions were the training sessions. The experiment was set up such that error augmentation subjects received the augmented error feedback during the training sessions, while the control subjects received the conventional feedback. Both groups received conventional feedback during the testing sessions to allow for statistical analysis.

\section{Data Analysis}

Time-to-target was the primary metric used to evaluate performance. Statistical analysis was performed using MATLAB [20] and STATISTICA [21]. Because a subject could overshoot the target, time-to-target was calculated as the time at which the displayed CoP indicator entered the target for the final time before the next target appeared. To eliminate anticipatory effects, only shifts away from center were considered. Reaction time was calculated by finding the time at which the subject began shifting and comparing it to the time at which the target appeared. Any shift with a negative reaction time was eliminated from consideration because the subject began shifting before the new target appeared. Any subject for whom over a third of the shifts within a single session were eliminated for this reason was removed from analysis. To allow for any residual effects of changing feedback between sessions to washout, only the final one-third of shifts within 
each session were considered. Finally, any time-to-target outliers outside of two mean absolute deviations of the subject's own shifts within the same session were eliminated.

An analysis of variance (ANOVA) test was applied to median time-to-target of each group and session for the remaining shifts to analyze any effects that the session number and feedback type had on the subjects' time-to-target during the testing sessions. The test involved two between-subject groups (control and error augmentation) and five within-subject session effects (testing sessions). The test considers how subjects in each group change over the course of the study and takes into account any interaction effects between the session number and the group type. A second ANOVA was performed on the two groups (control and error augmentation) across the four within-subject training sessions as well. Finally, to consider how individual testing sessions compare to each other across group types, a one-tailed Welch two sample t-test was performed on the median time-to-target data at each training or testing session.

\section{Results}

Data from one subject in the control group were removed from the analysis because that subject exhibited negative reaction times on more than one third of the shifts in the first testing session.

\section{ANOVA analyses}

As indicated in Table 1(A), there was a main effect as a result of the session number for the testing sessions in which standard feedback was given to both groups $(\mathrm{p} \simeq 0)$. Also, there was no main effect as the result of group type or the interaction between the group type and session number $(\mathrm{p} \geq 0.05)$. The subjects improved over the course of the study, but there was no clear difference in the improvement of final performance for each subject as a result of the different feedback types. This confirms the hypothesis that both groups improve their performance over the course of the study, but there is no difference in the total amount that they improve relative to their starting performance.

Moreover, Table 1(B) shows that for the training sessions, there was a main effect as a result of the group type, session number, and the interaction between the group type and session number ( $\mathrm{p} \simeq 0$ for all three). While the control subjects received the unaltered feedback during the train- 
ing sessions and the error augmentation subjects received the augmented feedback, both groups improved between the first and last sessions. These results are useful in visualizing how the error augmentation subjects are able to improve at a different rate, but the significant group effect was due largely to the fact that the two groups were receiving different feedback during these training sessions. However, this outcome confirms that error augmentation does promote improved performance across sessions even though it engenders performance that is quite different from that of the control group.

\subsection{Comparison between Groups}

To further examine any differences between the two feedback groups, a Welch two sample ttest was performed between the median time-to-target of each group for every session. While there was no significant difference for the first or last two testing sessions $(\mathrm{p} \geq 0.05)$, there was a significant difference for the third $(\mathrm{p}<0.05)$. This finding shows that the error augmentation group outperformed the control group during the third testing session, confirming the hypothesis that error augmentation may improve the temporary performance of the subjects even though there was no significant difference in the relative final steady state balance performance between the two groups. For the training sessions, there was a significant difference between groups for the first three sessions $(\mathrm{p} \simeq 0$ ), but no significant difference for the final session $(\mathrm{p} \geq 0.05)$.

\section{Discussion}

These results provide further evidence that varying visual feedback can have significant effects on balance performance $[6,8,12,22]$. Due to the simplicity of the weight shifting tasks, one might have expected subjects to have reached their peak performance by the end of the first few sessions. On the contrary, for subjects, particularly control subjects, performance generally improved with repetition after six sessions had been completed. This suggests that subjects were learning between the first and final sessions [14]. Eventually, subjects reached a steady state in their performance, but subjects experiencing error augmentation during the training sessions drove their weight shifting performance to this steady state faster. Error augmentation exaggerated deviations from the standard trajectory,

so the relative performance improvement may have come from generating more reliable shifts and 
reducing variability. Error augmentation, though, did not affect steady state time-to-target performance. As subjects are required to remain in the target for a specified duration, there is a practical limit on how quickly a shift can be completed. Therefore, it is not surprising that both groups reached a similar steady state performance. A possible limitation here is the lack of testing beyond the point at which subjects reached steady state performance. However, since error augmentation did not appear to affect the final level of steady state performance, it is not expected that there would be a difference in performance after an extended period. Nonetheless, a delayed retention test could be performed in future studies to analyze this aspect of performance.

When feedback differs between sensory systems, the central nervous system tends to rely on visual feedback, rather than the proprioceptive response, to correct the differences [23]. Therefore, it is expected that these results will vary as a result of changes in the error gain. Patton et al. compared the performance effects for error augmentation in arm-reaching tasks and found that increasing the gain improved performance effects [8]. In pilot studies, the error gain for balance tasks also affected performance effects, but there was a practical limit. In this study, only one error gain was selected because of the constraint on time to prevent fatigue. If the gain is decreased, it is less likely that results would be significant, since the augmented error visual feedback would become more similar to the control feedback. Meanwhile, since standing balance is intrinsically unstable, increasing the gain of feedback could theoretically cause instability, resulting in a fall. More realistically, subjects would likely decrease their reliance on the visual feedback [19], increasing their reliance on proprioception instead, negating any effects of altering the visual feedback [24, 25].

Changing the visual feedback between sessions likely kept the error augmentation subjects more engaged in the activity, which also could have contributed to performance effect. By receiving both error augmentation and unaltered feedback in alternating sessions, error augmentation subjects have an opportunity to "overlearn" during the testing sessions or are encouraged to be more engaged in the study. This could lead to improved performance after experiencing this test effect [26]. This hypothesis could be tested by including a third testing group that experiences a different change in visual feedback between tasks.

One extension of these results would be the application of error augmentation to stroke rehabilitation. Stroke can affect motor cortices of the brain, motor tracts, or pathways within the cerebrum 
and cerebellum $[27,28]$, and the loss of motor control diminishes balance in individuals with stroke. Stroke is the primary cause of falls that result in hospitalization for adults [3]. A loss of balance also limits daily activities and community participation for those with stroke [28]. Since lateral balance control provides a sensorimotor evaluation following stroke [2], the results of this study suggest that using error augmentation may maximize the impact of therapy in a short period of time. If error augmentation can help encourage patients to reach their maximum balance performance more quickly within a given session, the limited therapy time due to cost and fatigue can be used more effectively.

Before extension to therapy practice, these error augmentation results need to be verified in older, healthy subjects and in individuals with stroke. Unpublished results suggest that balance improvements due to changes in visual modalities translate across the different subject groups. It is therefore expected that the results found for this young, healthy group will likely hold for the older, healthy and stroke groups. Additionally, since balance abilities tend to deteriorate with age $[10,22]$, both a standard trajectory and an appropriate error gain will need to be determined for each group. Standard trajectories could be generated from new data or scaled from the trajectory herein according to differences in the average time-to-target. For individuals with stroke, the trajectory

could be modified to approach that of the age-matched healthy group as therapy progresses. If the average steady state time-to-target for these groups is worse than the young, healthy group, as unpublished results suggest, the current error augmentation path may also lead to improvements in overall performance.

\section{Acknowledgements}

This material is based upon work supported in part by the National Science Foundation under Grant no. IIS-1117706.

Thanks to Michael Villano, Aaron Striegel, Mitchell Kajzer, and Julaine Zenk for technical support and assistance with this study.

\section{Conflicts of Interest}

The authors have no conflict of interests to report related to this work. 
[1] E. Ravaioli, K. S. Oie, T. Kiemel, L. Chiari, and J. J. Jeka, "Nonlinear postural control in response to visual translation," Experimental Brain Research, vol. 160, no. 4, pp. 450-459, 2005.

[2] M. C. Dault, M. de Haart, A. C. Geurts, I. M. Arts, and B. Nienhuis, "Effects of visual center of pressure feedback on postural control in young and elderly healthy adults and in stroke patients," Human Movement Science, vol. 22, no. 3, pp. 221-236, 2003.

[3] R. A. Geiger, J. B. Allen, J. O’Keefe, and R. R. Hicks, "Balance and mobility following stroke: effects of physical therapy interventions with and without biofeedback/forceplate training," Physical Therapy, vol. 81, no. 4, pp. 995-1005, 2001.

[4] F. E. Huxham, P. A. Goldie, and A. E. Patla, "Theoretical considerations in balance assessment," Australian Journal of Physiotherapy, vol. 47, no. 2, pp. 89-100, 2001.

[5] R. Sigrist, G. Rauter, R. Riener, and P. Wolf, "Augmented visual, auditory, haptic, and multimodal feedback in motor learning: a review," Psychonomic Bulletin 6 Review, vol. 20, no. 1, pp. 21-53, 2013.

[6] M. W. Kennedy, C. R. Crowell, A. D. Striegel, M. Villano, and J. P. Schmiedeler, "Relative efficacy of various strategies for visual feedback in standing balance activities," Experimental Brain Research, vol. 230, no. 1, pp. 117-125, 2013.

[7] M. W. Kennedy, C. R. Crowell, M. Villano, and J. P. Schmiedeler, "Effects of filtering the center of pressure feedback provided in visually guided mediolateral weight shifting," PloS one, vol. 11, no. 3, p. e0151393, 2016.

[8] J. L. Patton, Y. J. Wei, P. Bajaj, and R. A. Scheidt, "Visuomotor learning enhanced by augmenting instantaneous trajectory error feedback during reaching," PloS One, vol. 8, no. 1, p. e46466, 2013.

[9] I. Sharp, F. Huang, and J. Patton, "Visual error augmentation enhances learning in three dimensions," Journal of Neuroengineering and Rehabilitation, vol. 8, no. 1, p. 1, 2011. 
[10] A. Zijlstra, M. Mancini, L. Chiari, and W. Zijlstra, "Biofeedback for training balance and mobility tasks in older populations: a systematic review," Journal of Neuroengineering and Rehabilitation, vol. 7, no. 1, p. 1, 2010.

[11] M. C. Kilby, S. M. Slobounov, and K. M. Newell, "Augmented feedback of COM and COP modulates the regulation of quiet human standing relative to the stability boundary," Gait $\&$ Posture, vol. 47, pp. 18-23, 2016.

[12] N. Vuillerme, R. Bertrand, and N. Pinsault, "Postural effects of the scaled display of visual foot center of pressure feedback under different somatosensory conditions at the foot and the ankle," Archives of Physical Medicine and Rehabilitation, vol. 89, no. 10, pp. 2034-2036, 2008.

[13] D. S. Nichols, "Balance retraining after stroke using force platform biofeedback," Physical Therapy, vol. 77, no. 5, pp. 553-558, 1997.

[14] R. A. Schmidt and T. Lee, Motor Control and Learning: A Behavioral Emphasis. Human Kinetics, 5 ed., 1988.

[15] M. W. Kennedy, J. P. Schmiedeler, C. R. Crowell, M. Villano, A. D. Striegel, and J. Kuitse, "Enhanced feedback in balance rehabilitation using the Nintendo Wii balance board," in e-Health Networking Applications and Services (Healthcom), 2011 13th IEEE International Conference on, pp. 162-168, IEEE, 2011.

[16] R. A. Clark, A. L. Bryant, Y. Pua, P. McCrory, K. Bennell, and M. Hunt, "Validity and reliability of the Nintendo Wii balance board for assessment of standing balance," Gait \& Posture, vol. 31, no. 3, pp. 307-310, 2010.

[17] W.-D. Chang, W.-Y. Chang, C.-L. Lee, and C.-Y. Feng, "Validity and reliability of Wii Fit Balance Board for the assessment of balance of healthy young adults and the elderly," Journal of Physical Therapy Science, vol. 25, no. 10, pp. 1251-1253, 2013.

[18] M. W. Kennedy, T. Bretl, and J. P. Schmiedeler, "Interpreting lateral dynamic weight shifts using a simple inverted pendulum model," Gait \& Posture, vol. 40, no. 1, pp. 134-139, 2014. 
[19] P. Rougier, "Visual feedback induces opposite effects on elementary centre of gravity and centre of pressure minus centre of gravity motions in undisturbed upright stance," Clinical Biomechanics, vol. 18, no. 4, pp. 341-349, 2003.

[20] M. Inc., MATLAB and Statistics Toolbox Release 2014a. Natick, Massachusetts, United States, 2014.

[21] I. StatSoft, STATISTICA 12. Dell Software, 2013.

[22] H. Bateni, "Changes in balance in older adults based on use of physical therapy vs the Wii Fit gaming system: a preliminary study," Physiotherapy, vol. 98, no. 3, pp. 211-216, 2012.

[23] C. S. Harris, "Perceptual adaptation to inverted, reversed, and displaced vision.," Psychological Review, vol. 72, no. 6, p. 419, 1965.

[24] R. Peterka, "Sensorimotor integration in human postural control," Journal of Neurophysiology, vol. 88 , no. 3, pp. 1097-1118, 2002.

[25] S.-J. Kim, M. Ogilvie, N. Shimabukuro, T. Stewart, and J.-H. Shin, "Effects of visual feedback distortion on gait adaptation: Comparison of implicit visual distortion versus conscious modulation on retention of motor learning," IEEE Transactions on Biomedical Engineering, vol. 62, no. 9, pp. 2244-2250, 2015.

[26] H. L. Roediger and J. D. Karpicke, "The power of testing memory: Basic research and implications for educational practice," Perspectives on Psychological Science, vol. 1, no. 3, pp. 181-210, 2006.

[27] L. Blum and N. Korner-Bitensky, "Usefulness of the Berg Balance Scale in stroke rehabilitation: a systematic review," Physical Therapy, vol. 88, no. 5, pp. 559-566, 2008.

[28] P. Langhorne, F. Coupar, and A. Pollock, "Motor recovery after stroke: a systematic review," The Lancet Neurology, vol. 8, no. 8, pp. 741-754, 2009. 


\section{List of Figures}

1 Experimental Setup. Each subject places one foot on each board, facing the large monitor. The boards connect wirelessly to the laptop running the software, which is not visible to the subject. . . . . . . . . . . . . . . . . . . . .

2 Error augmentation feedback generation. (A) Trajectory data from a total of 2287 lateral shifts that 27 young, healthy subjects performed in a previous study [6]. Each blue line represents an individual shift. The pink trajectories represent the analysis from all of the individual trajectories combined. The median trajectory is solid, while the interquartile positions are indicated by the dotted lines. These results demonstrate that there is a standard trajectory that most subjects follow while shifting to the target. (B) Error augmentation calculation. Using the standard trajectory, the error of the true $\mathrm{CoP}$ is calculated. A gain is then applied to the error and added to the true CoP to generate the displayed CoP feedback in real time. . .

3 Time-To-Target for Error Augmentation Subjects. The median time-to-target improves between each session (3A) until reaching steady state performance by the third training session (fifth session overall). Error augmentation was applied during the even-numbered sessions $(3 \mathrm{~B}) \ldots \ldots \ldots \ldots \ldots$ 


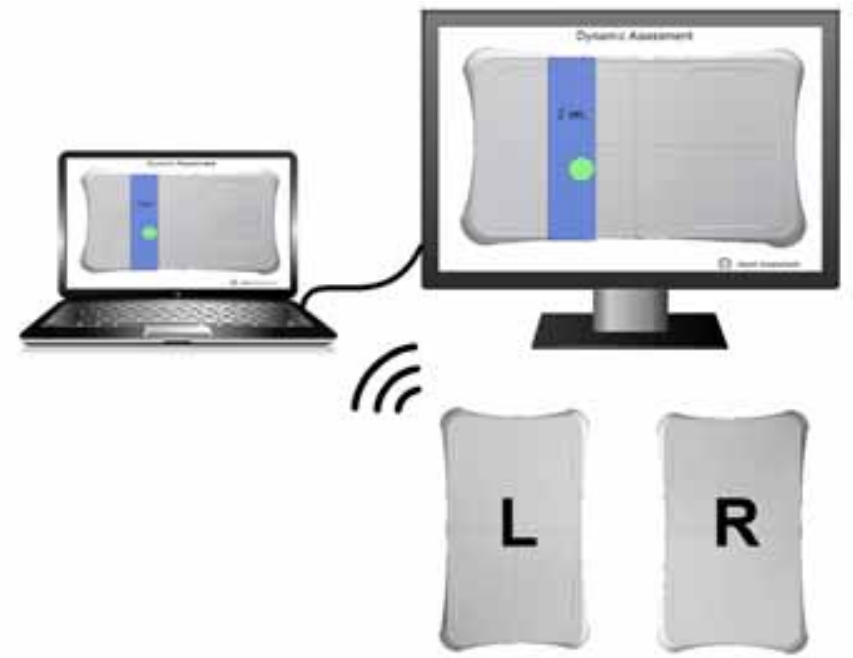

Figure 1: Experimental Setup. Each subject places one foot on each board, facing the large monitor. The boards connect wirelessly to the laptop running the software, which is not visible to the subject. 


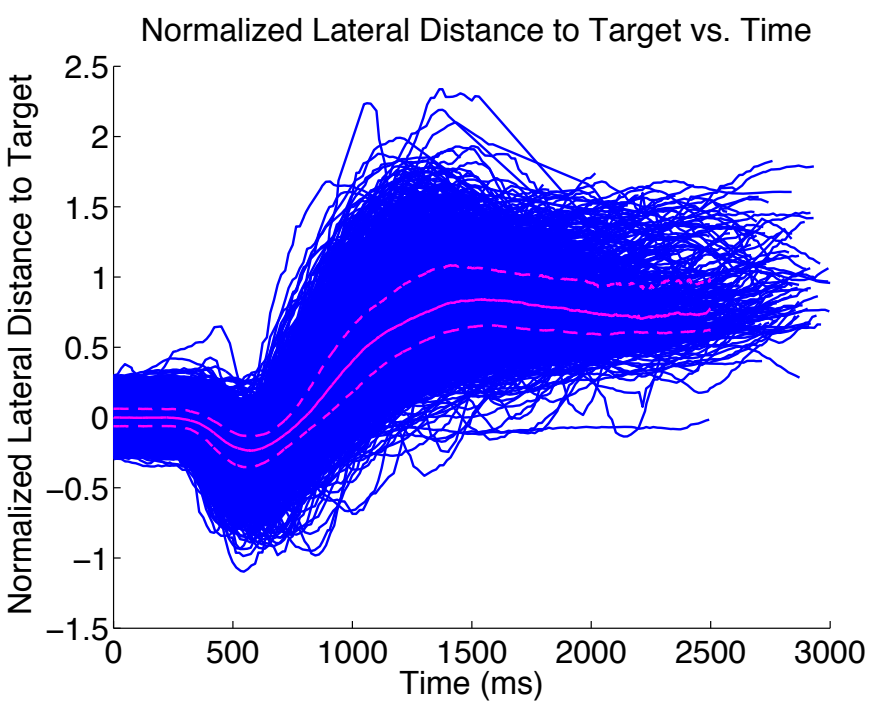

A

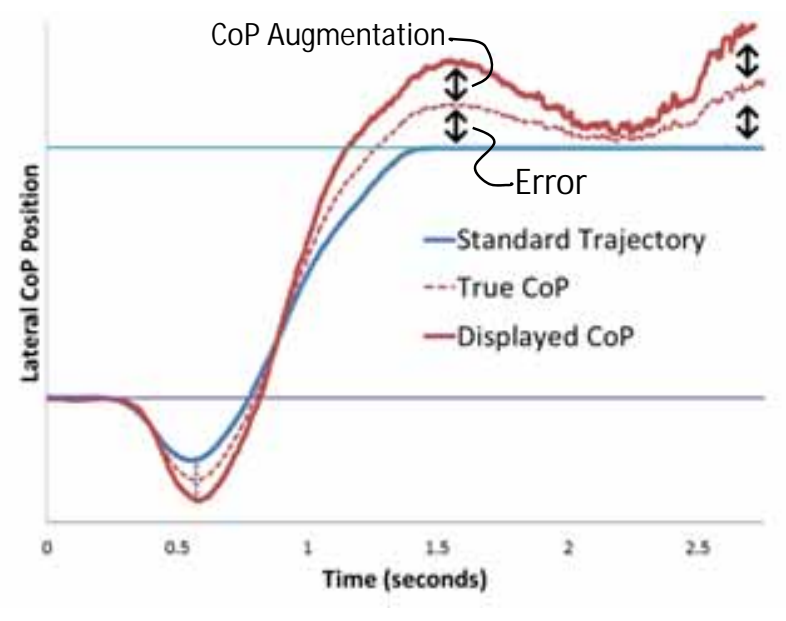

$\mathrm{B}$

Figure 2: Error augmentation feedback generation. (A) Trajectory data from a total of 2287 lateral shifts that 27 young, healthy subjects performed in a previous study [6]. Each blue line represents an individual shift. The pink trajectories represent the analysis from all of the individual trajectories combined. The median trajectory is solid, while the interquartile positions are indicated by the dotted lines. These results demonstrate that there is a standard trajectory that most subjects follow while shifting to the target. (B) Error augmentation calculation. Using the standard trajectory, the error of the true $\mathrm{CoP}$ is calculated. A gain is then applied to the error and added to the true $\mathrm{CoP}$ to generate the displayed $\mathrm{CoP}$ feedback in real time. 


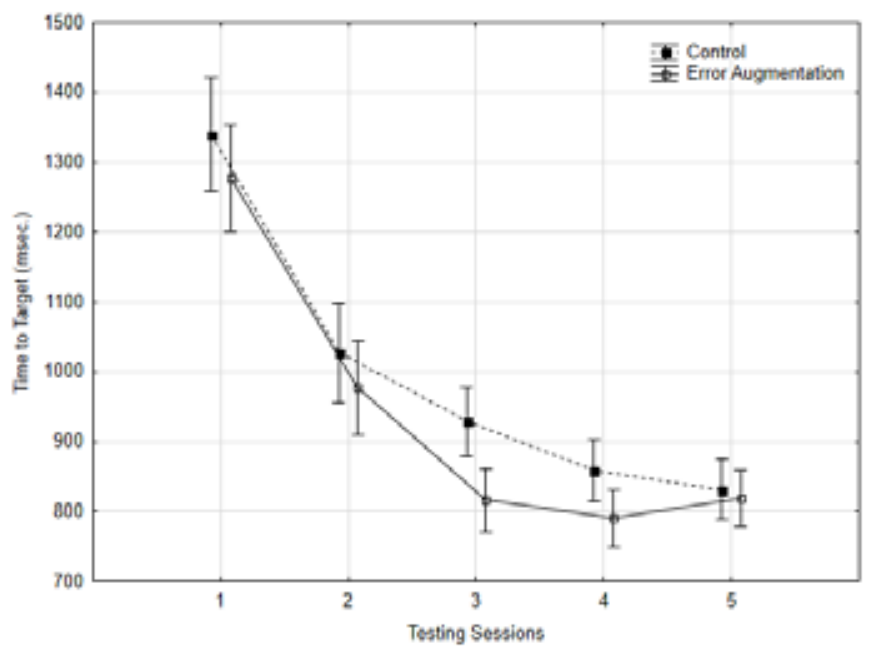

A

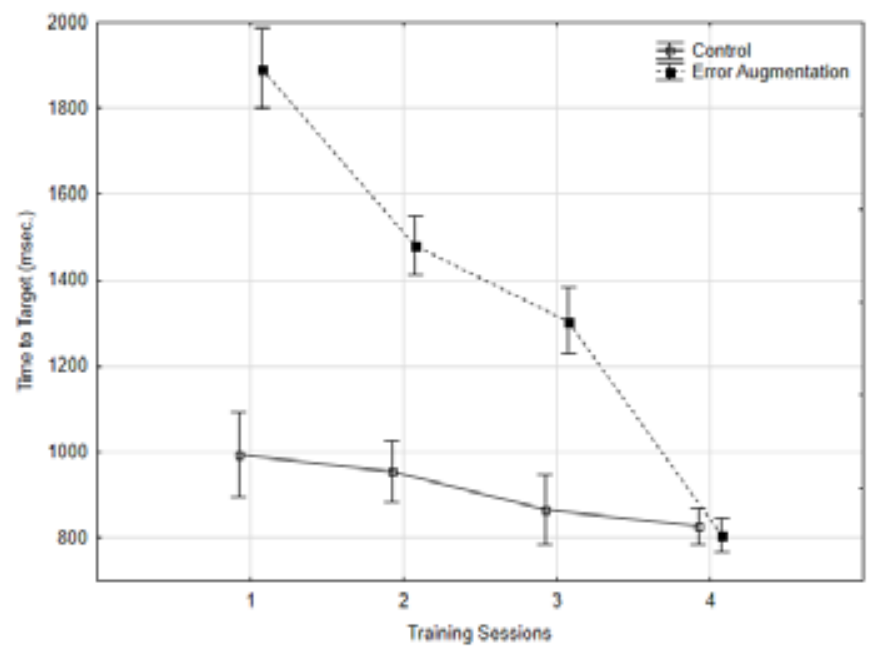

$\mathrm{B}$

Figure 3: Time-To-Target for Error Augmentation Subjects. The median time-to-target improves between each session (3A) until reaching steady state performance by the third training session (fifth session overall). Error augmentation was applied during the even-numbered sessions (3B). 


\section{List of Tables}

1 Repeated Measures Analysis of Variance. There is an effect due to the session number during the testing sessions but no group or interaction effect, demonstrating that the subjects are improving over the course of the study, but do not improve their final performance of the task relative to each other (Table 1(A)). Meanwhile, the group, session, and interaction effects demonstrate a significant difference in performance for the error augmentation subjects during the training sessions (Table 1(B)). . . . 
Table 1: Repeated Measures Analysis of Variance. There is an effect due to the session number during the testing sessions but no group or interaction effect, demonstrating that the subjects are improving over the course of the study, but do not improve their final performance of the task relative to each other (Table 1(A)). Meanwhile, the group, session, and interaction effects demonstrate a significant difference in performance for the error augmentation subjects during the training sessions (Table 1(B)).

(A) Testing Sessions

\begin{tabular}{lrrr}
\hline Effect & DoF & $\mathrm{F}$ & $\mathrm{p}$-value \\
\hline Intercept & 1 & 1209.857 & ${ }^{*} 0.000$ \\
Group & 1 & 1.213 & 0.279 \\
Error & 30 & & \\
\hline Sessions & 4 & 36.344 & ${ }^{*} 0.000$ \\
Sessions $^{*}$ Group & 4 & 0.281 & 0.890 \\
Error & 120 & & \\
\hline
\end{tabular}

(B) Training Sessions

\begin{tabular}{lrrr}
\hline Effect & DoF & $\mathrm{F}$ & $\mathrm{p}$-value \\
\hline Intercept & 1 & 959.221 & ${ }^{*} 0.000$ \\
Group & 1 & 39.148 & ${ }^{*} 0.000$ \\
Error & 32 & & \\
\hline Sessions & 4 & 37.458 & ${ }^{*} 0.000$ \\
Sessions ${ }^{*}$ Group & 4 & 19.491 & ${ }^{*} 0.000$ \\
Error & 96 & & \\
\hline
\end{tabular}

